\title{
Adomian Decomposition Approach to the Solution of the Burger's Equation
}

\author{
Iyakino P. Akpan \\ Department of Basic Science, College of Agriculture, Lafia, Nigeria \\ Email: akpanip@yahoo.com
}

Received 16 July 2015; accepted 6 September 2015; published 9 September 2015

Copyright (C) 2015 by author and Scientific Research Publishing Inc.

This work is licensed under the Creative Commons Attribution International License (CC BY). http://creativecommons.org/licenses/by/4.0/

c) (i) Open Access

\begin{abstract}
Adomian decomposition method is presented as a method for the solution of the Burger's equation, a popular PDE model in the fluid mechanics. The method is computationally simple in application. The approximate solution is obtained by considering only the first two terms of the decomposition in this paper. Numerical experimentation shows accuracy of a minimum error of order five for various space steps and coefficient of kinematic viscosity. The method is considered high in accuracy.
\end{abstract}

\section{Keywords}

Adomian Decomposition, Burger's Equation, Kinematic Viscosity, Nonlinear Operators

\section{Introduction}

Burger's equation is a fundamental partial differential equation in fluid mechanics. It is also a very important model encountered in several areas of applied mathematics such as heat conduction, acoustic waves, gas dynamics and traffic flow [1]. Analytical solutions of the partial differential equations modeling physical phenomena exist only in few of the cases. Therefore the need for the construction of efficient numerical methods for the approximate solution of these models always exists. Many of the analytical solutions to the Burger's equation involve Fourier series. According to [2], the convergence of such Fourier-series based solutions is very slow. Several researchers have proposed various numerical methods for the solution of the Burger's equation. [3] and [4] used the finite element method for the solution of the Burger's equation. [5] and [6] used the finite difference method. [7] used the direct variational method while [8] used the projection method by B-spline.

A decomposition method which provides convergent solutions to nonlinear stochastic operator equations was developed in [9]. [10] gave proof of convergence of Adomian decomposition method when applied to differential equations. [11] constructed an algorithm for solving nonlinear equations based on Newton-Raphson method 
using Adomian decomposition approach. In an effort to extend the usefulness of Adomian's decomposition method into other areas of mathematics, [12] developed a technique for calculating Adomian's polynomials for nonlinear operators. [13] presented a method for the solution of homogeneous differential equations using Adomian's decomposition without noisy terms. [14] extended the application of the Adomian decomposition method for the calculations of non-differential functions.

In this paper, presentation of a numerical method for the solution of the nonlinear reaction-diffusion Burger's equation using Adomian decomposition is made. The organization of this paper is as follows: in Section 2 the theoretical approach is presented. In Section 3 the Adomian's polynomials for the Burger's equation are determined. In Section 4 computational results for the Burger's equation using Adomian's decomposition are presented while conclusion is presented in Section 5.

\section{Theoretical Approach}

Consider the Burger's equation

$$
\frac{\partial u}{\partial t}+u \frac{\partial u}{\partial x}=\varepsilon \frac{\partial^{2} u}{\partial x^{2}}, 0 \leq x \leq 1,0 \leq t \leq T
$$

Subject to initial condition

$$
u(x, 0)=f(x)
$$

And boundary conditions:

$$
\begin{aligned}
& u(0, t)=g_{1}(t) \\
& u(1, t)=g_{2}(x)
\end{aligned}
$$

Defining the operators:

$$
L_{t}=\frac{\partial}{\partial t}, L_{x}=\frac{\partial^{2}}{\partial x^{2}}, N u=u \frac{\partial u}{\partial x}
$$

Equation (1) can be written as:

$$
L_{t} u+N u=\varepsilon L_{x} u
$$

Solving Equation (6) for $L_{t}$ and $L_{x}$ respectively gives

$$
L_{t} u=\varepsilon L_{x} u-N u
$$

and

$$
L_{x} u=\varepsilon^{-1}\left(L_{t} u+N u\right)
$$

where $L_{t}^{-1}$ and $L_{x}^{-1}$ are the inverse operators of $L_{t}$ and $L_{x}$ given respectively as:

$$
L_{t}^{-1}=\int(.) \mathrm{d} t \text { and } L_{x}^{-1}=\iint(.) \mathrm{d} x
$$

Operating both sides of Equations (7) and (8) with the inverse operators (9) we obtain:

$$
\begin{gathered}
u=\varphi_{0}+L_{t}^{-1}\left(\varepsilon \frac{\partial^{2} u}{\partial x^{2}}-u \frac{\partial u}{\partial x}\right) \\
u=\omega_{0}+L_{x}^{-1} \varepsilon^{-1}\left(\frac{\partial u}{\partial t}+u \frac{\partial u}{\partial x}\right)
\end{gathered}
$$

where

$$
\varphi_{0}=u(x, 0)
$$

and

$$
\omega_{0}=u(x, 0)+x \frac{\partial u(x, 0)}{\partial x}
$$


Adding Equations (10) and (11) and dividing by 2, gives the canonical form:

$$
u=\frac{1}{2}\left[\left(\varphi_{0}+\omega_{0}\right)+L_{t}^{-1}\left(\varepsilon \frac{\partial^{2} u}{\partial x^{2}}-u \frac{\partial u}{\partial x}\right)+\varepsilon^{-1} L_{x}^{-1}\left(\frac{\partial u}{\partial t}+u \frac{\partial u}{\partial x}\right)\right]
$$

The parameterized form of (14) is

$$
u=u_{0}+\frac{\lambda}{2}\left[\varepsilon^{-1} L_{x}^{-1}\left(\frac{\partial u}{\partial t}+u \frac{\partial u}{\partial x}\right)+L_{t}^{-1}\left(\varepsilon \frac{\partial^{2} u}{\partial x^{2}}-u \frac{\partial u}{\partial x}\right)\right]
$$

where

$$
u_{0}=\frac{1}{2}\left(\varphi_{0}+\omega_{0}\right)
$$

According to [15] the parameterized decomposition forms of $u$ and $N_{u}$ are:

$$
\begin{gathered}
u=\sum_{n=0}^{\infty} \lambda^{n} u_{n} \\
u \frac{\partial u}{\partial x}=N u=\sum_{n=0}^{\infty} \lambda^{n} A_{n}
\end{gathered}
$$

$A_{n}$ are the Adomian's polynomials which can be generated for all types of nonlinearities [16].

Substituting Equations (17) and (18) into Equation (15) gives

$$
\sum_{n=0}^{\infty} \lambda^{n} u_{n}=u_{0}+\frac{\lambda}{2}\left[\varepsilon^{-1} L_{x}^{-1}\left(\frac{\partial}{\partial t}\left(\sum_{n=0}^{\infty} \lambda^{n} u_{n}\right)+\sum_{n=0}^{\infty} \lambda^{n} A^{n}\right)+L_{t}^{-1}\left(\varepsilon \frac{\partial^{2}}{\partial x^{2}}\left(\sum_{n=0}^{\infty} \lambda^{n} u_{n}\right)-\sum_{n=0}^{\infty} \lambda^{n} A^{n}\right)\right]
$$

Expanding both sides of Equation (19) gives

$$
\begin{aligned}
\lambda^{0} u_{0}+\lambda^{1} u_{1}+\lambda^{2} u_{2}+\lambda^{3} u_{3}+\lambda^{4} u_{4}+\lambda^{5} u_{5}+\cdots \\
=u_{0}+\frac{\lambda}{2}\left[\varepsilon ^ { - 1 } L _ { x } ^ { - 1 } \left(\lambda^{0} \frac{\partial u_{0}}{\partial t}+\lambda^{1} \frac{\partial u_{1}}{\partial t}+\lambda^{2} \frac{\partial u_{2}}{\partial t}+\lambda^{3} \frac{\partial u_{3}}{\partial t}+\lambda^{4} \frac{\partial u_{4}}{\partial t}+\lambda^{5} \frac{\partial u_{5}}{\partial t}\right.\right. \\
\left.+\cdots+\lambda^{0} A_{0}+\lambda^{1} A_{1}+\lambda^{2} A_{2}+\lambda^{3} A_{3}+\lambda^{4} A_{4}+\lambda^{5} A_{5}+\cdots\right) \\
+\varepsilon L_{t}^{-1}\left(\lambda^{0} \frac{\partial^{2} u_{0}}{\partial x^{2}}+\lambda^{0} \frac{\partial^{2} u_{0}}{\partial x^{2}}+\lambda^{0} \frac{\partial^{2} u_{0}}{\partial x^{2}}+\lambda^{0} \frac{\partial^{2} u_{0}}{\partial x^{2}}+\lambda^{0} \frac{\partial^{2} u_{0}}{\partial x^{2}}\right. \\
\left.\left.+\lambda^{0} \frac{\partial^{2} u_{0}}{\partial x^{2}}+\cdots-\left(\lambda^{0} A_{0}+\lambda^{1} A_{1}+\lambda^{2} A_{2}+\lambda^{3} A_{3}+\lambda^{4} A_{4}+\lambda^{5} A_{5}+\cdots\right)\right)\right]
\end{aligned}
$$

By comparing coefficients of both hand sides of Equation (20), it is obtained that:

$$
\begin{aligned}
& u_{1}=\frac{1}{2}\left[\varepsilon^{-1} L_{x}^{-1}\left(\frac{\partial u_{0}}{\partial t}+A_{0}\right)+L_{t}^{-1}\left(\varepsilon \frac{\partial^{2} u_{0}}{\partial x^{2}}-A_{0}\right)\right] \\
& u_{2}=\frac{1}{2}\left[\varepsilon^{-1} L_{x}^{-1}\left(\frac{\partial u_{1}}{\partial t}+A_{1}\right)+L_{t}^{-1}\left(\varepsilon \frac{\partial^{2} u_{1}}{\partial x^{2}}-A_{1}\right)\right] \\
& u_{3}=\frac{1}{2}\left[\varepsilon^{-1} L_{x}^{-1}\left(\frac{\partial u_{2}}{\partial t}+A_{2}\right)+L_{t}^{-1}\left(\varepsilon \frac{\partial^{2} u_{2}}{\partial x^{2}}-A_{2}\right)\right] \\
& \vdots \\
& u_{n}=\frac{1}{2}\left[\varepsilon^{-1} L_{x}^{-1}\left(\frac{\partial u_{n-1}}{\partial t}+A_{n-1}\right)+L_{t}^{-1}\left(\varepsilon \frac{\partial^{2} u_{n-1}}{\partial x^{2}}-A_{n-1}\right)\right]
\end{aligned}
$$


From which we establish the recursive relation

$$
u_{n+1}=\frac{1}{2}\left[\varepsilon^{-1} L_{x}^{-1}\left(\frac{\partial u_{n}}{\partial t}+A_{n}\right)+L_{t}^{-1}\left(\varepsilon \frac{\partial^{2} u_{n}}{\partial x^{2}}-A_{n}\right)\right]
$$

\section{Determination of Adomian's Polynomials}

The Adomian's polynomials, $A_{n}$ are defined in such a way that

$$
A_{0}=A_{0}\left(u_{0}\right), A_{1}=A_{1}\left(u_{0}, u_{1}\right), A_{2}=A_{2}\left(u_{0}, u_{1}, u_{2}\right), \cdots, A_{n}\left(u_{0}, u_{1}, u_{2}, \cdots, u_{n}\right)
$$

Substituting Equation (17) into Equation (18) and expanding the RHS gives

$$
\begin{aligned}
N u= & u_{0} \frac{\partial u_{0}}{\partial x}+\lambda\left(u_{0} \frac{\partial u_{1}}{\partial x}+u_{1} \frac{\partial u_{0}}{\partial x}\right)+\lambda^{2}\left(u_{0} \frac{\partial u_{2}}{\partial x}+u_{1} \frac{\partial u_{1}}{\partial x}+u_{2} \frac{\partial u_{0}}{\partial x}\right) \\
& +\lambda^{3}\left(u_{0} \frac{\partial u_{3}}{\partial x}+u_{1} \frac{\partial u_{2}}{\partial x}+u_{2} \frac{\partial u_{1}}{\partial x} u_{3} \frac{\partial u_{0}}{\partial x}\right) \\
& +\lambda^{4}\left(u_{0} \frac{\partial u_{4}}{\partial x}+u_{1} \frac{\partial u_{3}}{\partial x}+u_{2} \frac{\partial u_{2}}{\partial x}+u_{3} \frac{\partial u_{1}}{\partial x}+u_{4} \frac{\partial u_{0}}{\partial x}\right) \\
& +\lambda^{5}\left(u_{0} \frac{\partial u_{5}}{\partial x}+u_{1} \frac{\partial u_{4}}{\partial x}+u_{2} \frac{\partial u_{3}}{\partial x}+u_{3} \frac{\partial u_{2}}{\partial x}+u_{4} \frac{\partial u_{1}}{\partial x}+u_{5} \frac{\partial u_{0}}{\partial x}\right)+\cdots
\end{aligned}
$$

From Equation (22), we establish that the Adomian's polynomials have the following forms:

$$
\begin{aligned}
& A_{0}=u_{0} \frac{\partial u_{0}}{\partial x} \\
& A_{1}=u_{0} \frac{\partial u_{1}}{\partial x}+u_{1} \frac{\partial u_{0}}{\partial x} \\
& A_{2}=u_{0} \frac{\partial u_{2}}{\partial x}+u_{1} \frac{\partial u_{1}}{\partial x}+u_{2} \frac{\partial u_{0}}{\partial x} \\
& A_{3}=u_{0} \frac{\partial u_{3}}{\partial x}+u_{1} \frac{\partial u_{2}}{\partial x}+u_{2} \frac{\partial u_{1}}{\partial x} u_{3} \frac{\partial u_{0}}{\partial x} \\
& A_{4}=u_{0} \frac{\partial u_{4}}{\partial x}+u_{1} \frac{\partial u_{3}}{\partial x}+u_{2} \frac{\partial u_{2}}{\partial x}+u_{3} \frac{\partial u_{1}}{\partial x}+u_{4} \frac{\partial u_{0}}{\partial x} \\
& A_{5}=u_{0} \frac{\partial u_{5}}{\partial x}+u_{1} \frac{\partial u_{4}}{\partial x}+u_{2} \frac{\partial u_{3}}{\partial x}+u_{3} \frac{\partial u_{2}}{\partial x}+u_{4} \frac{\partial u_{1}}{\partial x}+u_{5} \frac{\partial u_{0}}{\partial x}
\end{aligned}
$$

The Adomian's polynomials for Equation (1) are obtained from the recurrent relation

$$
A_{n}=\sum_{\substack{0 \leq i \leq n \\ n \geq j \geq 0}} u_{i} \frac{\partial u_{j}}{\partial x}
$$

\section{Numerical Experiment}

We present numerical results to illustrate the effectiveness of the proposed method. Consider Burger's Equation (1) with the following initial and boundary conditions.

$$
\begin{gathered}
u(x, 0)=\frac{2 \varepsilon \pi \sin (\pi x)}{a+\cos (\pi x)} \\
u(0, t)=u(x, 1)=0
\end{gathered}
$$


The exact solution of Equation (1) with the above conditions was given in [1] as

$$
u(x, t)=\frac{2 \varepsilon \pi \mathrm{e}^{-\pi^{2} \varepsilon t} \sin (\pi x)}{a+\pi \mathrm{e}^{-\pi^{2} \varepsilon t} \cos (\pi x)}
$$

\section{Computations of Absolute and Relative Errors}

Absolute errors of the method were computed by use of the formula:

$$
\left|u_{i j}-u\left(x_{i}, t_{j}\right)\right|
$$

where the numerical solution at the grid point $\left(x_{i}, t_{j}\right)$ is $u_{i j}$, and the exact solution at the same grid point is $u\left(x_{i}, t_{j}\right)$.

Relative errors were computed by use of the formula:

$$
\left|\frac{u_{i j}-u\left(x_{i}, t_{j}\right)}{1+u\left(x_{i}, t_{j}\right)}\right|
$$

where the numerical solution at the grid point $\left(x_{i}, t_{j}\right)$ is $u_{i j}$, and the exact solution at the same grid point is $u\left(x_{i}, t_{j}\right)$.

Figure 1 is a 3 dimensional plot of the surface $u(x, t)=\frac{2 \varepsilon \pi \mathrm{e}^{-\pi^{2} \varepsilon t} \sin (\pi x)}{a+\pi \mathrm{e}^{-\pi^{2} \varepsilon t} \cos (\pi x)}$ for $0 \leq x \leq 1,0 \leq t \leq 1, a=2$, $\varepsilon=0.001$, while Figure 2 is a 3 dimensional plot of the surface $u(x, t)=\frac{2 \varepsilon \pi \mathrm{e}^{-\pi^{2} \varepsilon t} \sin (\pi x)}{a+\pi \mathrm{e}^{-\pi^{2} \varepsilon t} \cos (\pi x)}$ for $0 \leq x \leq 1$, $0 \leq t \leq 1, a=2, \varepsilon=0.01$.

\section{Conclusion}

In this paper, Adomian decomposition method is used to solve the burger's equation numerically. From Table 1 and Table 2, this method is considered to give accurate results for specified values of the parameter, $\varepsilon$. We considered the first two terms of the decomposition to make the approximate solution. Accuracy of the method

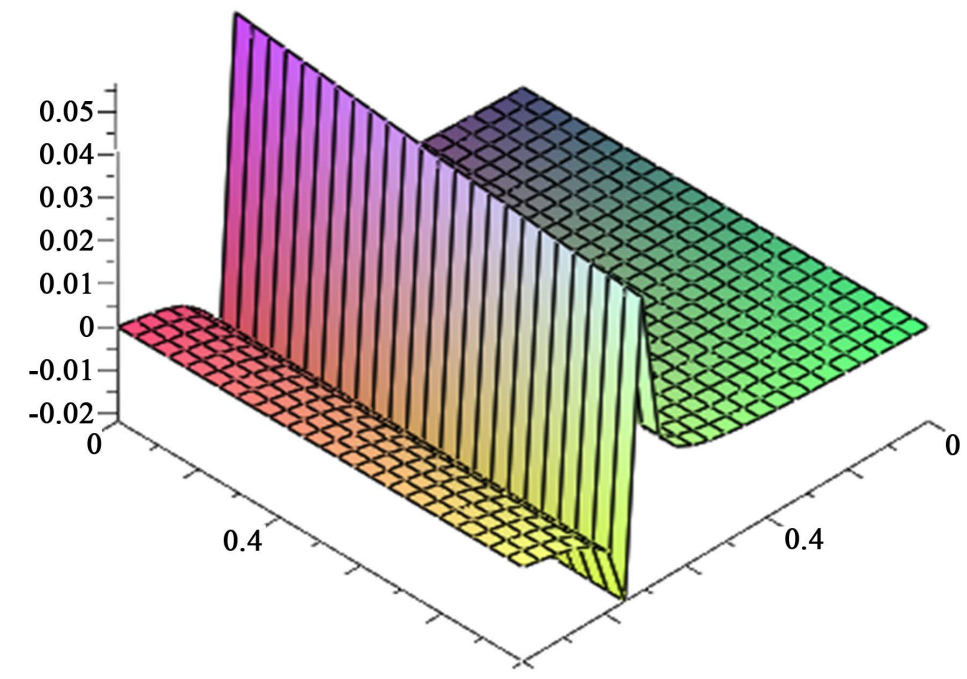

Figure 1. A 3 dimensional plot of the surface $u(x, t)=\frac{2 \varepsilon \pi \mathrm{e}^{-\pi^{2} \varepsilon t} \sin (\pi x)}{a+\pi \mathrm{e}^{-\pi^{2} \varepsilon t} \cos (\pi x)}$ for $0 \leq x \leq 1,0 \leq t \leq 1, a=2, \varepsilon=0.001$. 


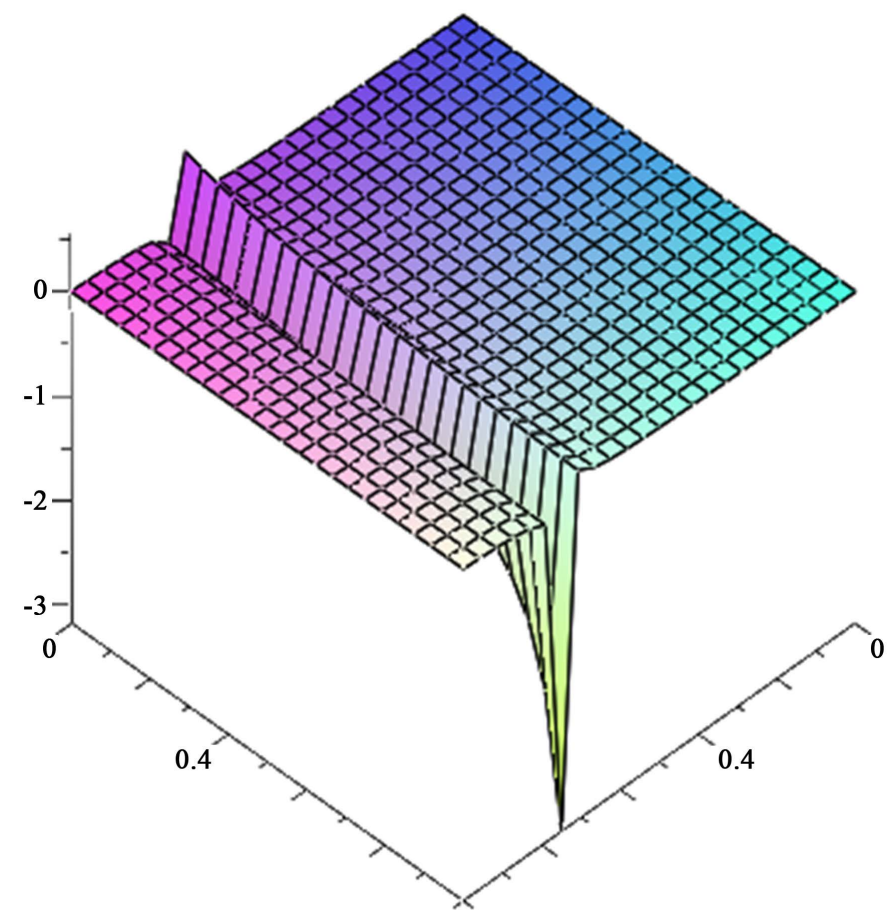

Figure 2. A 3 dimensional plot of the surface $u(x, t)=\frac{2 \varepsilon \pi \mathrm{e}^{-\pi^{2} \varepsilon t} \sin (\pi x)}{a+\pi \mathrm{e}^{-\pi^{2} \varepsilon t} \cos (\pi x)}$ for $0 \leq x \leq 1,0 \leq t \leq 1, a=2, \varepsilon=0.01$.

Table 1. Approximate solutions by Adomian's decomposition, errors and relative errors for $t=0.2, \varepsilon=0.001, a=2$.

\begin{tabular}{ccccc}
\hline$x$ & Adomian & Theoretical solution & Error & Relative Errors \\
\hline 0.1 & 0.0006610924400 & 0.0003891849100 & $2.719075297 \times 10^{-4}$ & $2.718017485 \times 10^{-4}$ \\
0.2 & 0.0013184569550 & 0.0008129882596 & $5.054686954 \times 10^{-4}$ & $5.050580892 \times 10^{-4}$ \\
0.3 & 0.001968724990 & 0.0013210998120 & $6.47625178 \times 10^{-4}$ & $6.467707292 \times 10^{-4}$ \\
0.4 & 0.002596743099 & 0.0020106848070 & $5.86058292 \times 10^{-4}$ & $5.84882278 \times 10^{-4}$ \\
0.5 & 0.0031486447293 & 0.0031366545250 & $1.9922768 \times 10^{-5}$ & $1.986047255 \times 10^{-5}$ \\
0.6 & 0.003546100003 & 0.005799752849 & $2.253652846 \times 10^{-3}$ & $2.240657585 \times 10^{-3}$ \\
0.7 & 0.003616277199 & 0.03291862860 & $2.930235148 \times 10^{-2}$ & $2.836849937 \times 10^{-2}$ \\
0.8 & 0.003425426033 & -0.006782588856 & $1.020801489 \times 10^{-2}$ & $1.027772447 \times 10^{-2}$ \\
\hline
\end{tabular}

Table 2. Approximate solutions by Adomian's decomposition, errors and relative errors for $t=0.05, \varepsilon=0.01, a=2$.

\begin{tabular}{ccccc}
\hline$x$ & Adomian & Theoretical Solution & Error & Relative Errors \\
\hline 0.2 & 0.01342133395 & 0.008119261273 & $5.302072677 \times 10^{-3}$ & $5.259370475 \times 10^{-3}$ \\
0.4 & 0.02618512105 & 0.02006671613 & $6.11840492 \times 10^{-3}$ & $5.998043877 \times 10^{-3}$ \\
0.6 & 0.03577004231 & 0.05766474009 & $2.189469778 \times 10^{-2}$ & $2.070098109 \times 10^{-2}$ \\
0.8 & 0.03132012403 & -0.06901554553 & $1.003356696 \times 10^{-1}$ & $1.077737325 \times 10^{-1}$ \\
\hline
\end{tabular}


can be improved by considering more terms in the solution approximation. All computations were carried out using Maple 15.

\section{References}

[1] Maleknejad, K., Babolian, E., Shaerlar, A.J. and Jahnagir, M. (2011) Operational Matrices for Solving Burgers Equation by Using Block-Pulse Functions with Error Analysis. Australian Journal of Basic and Applied Sciences, 5, 602609.

[2] Aksan, E.N., Ozdes, A. and Ozia, T. (2006) A Numerical Solution of Burger's Based Squares Approximation. Applied Mathematics and Computation, 176, 270-279. http://dx.doi.org/10.1016/j.amc.2005.09.045

[3] Varoglu, E. and Finn, W.D.L. (1980) Space-Time Finite Elements Incorporating Characteristics for Burger’s Equation. International Journal for Numerical Methods in Engineering, 16, 171-184. http://dx.doi.org/10.1002/nme.1620160112

[4] Caldwell, J., Wanless, P. and Cook, A.E. (1981) A Finite Element Approach to Burger’s Equation. Applied Mathematical Modelling, 5, 189-193. http://dx.doi.org/10.1016/0307-904X(81)90043-3

[5] Smith, G.D. (1989) Numerical Solution of Partial Differential Equations. Oxford Press, Oxford.

[6] Ames, W.F. (1992) Numerical Methods for Partial Differential Equations. Academic Press, New York.

[7] Ozas, T. and Ozdes, A. (1996) A Direct Variational Method Applied to Burgers Equation. Journal of Computational and Applied Mathematics, 71, 163-175. http://dx.doi.org/10.1016/0377-0427(95)00221-9

[8] Dag, J., Saka, B. and Boz, A. (2005) B-Splin Galekin Method for Numerical Solutions of the Burgers Equation. Applied Mathematics and Computation, 166, 506-522. http://dx.doi.org/10.1016/j.amc.2004.06.078

[9] Adomian, G. (1986) Nonlinear Stochastic Operator Equations. Academic Press, New York.

[10] Abbaoui, K. and Cherruault, Y. (1994) Convergence of Adomian’s Method Applied to Differential Equations. Computers \& Mathematics with Applications, 28, 103-109. http://dx.doi.org/10.1016/0898-1221(94)00144-8

[11] Abbasbandy, S. (2003) Improving Newton-Raphson Method for Nonlinear Equations by Modified Adomian Decomposition Method. Applied Mathematics and Computation, 145, 887-893. http://dx.doi.org/10.1016/S0096-3003(03)00282-0

[12] Chen, W. and Lu, Z. (2004) An Algorithm for Adomian Decomposition Method. Applied Mathematics and Computation, 170, 570-583. http://dx.doi.org/10.1016/j.amc.2003.10.037

[13] Gharsseldien, Z.M. and Hemida, K. (2009) New Technique to Avoid Noise Terms on the Solution of Inhomogeneous Differential Equations by Using Adomian Decomposition Method. Communication in Nonlinear Science and Numerical Simulation, 14, 685-696. http://dx.doi.org/10.1016/j.cnsns.2007.11.018

[14] Wu, G.C. (2011) Adomian Decomposition Method for Non-Smooth Initial Value Problems. Mathematical and Computer Modeling, 54, 2104-2108. http://dx.doi.org/10.1016/j.mcm.2011.05.018

[15] Mamaloukas, C. (2000) An Approximate Solution of Burgers Equation Using Adomian's Decomposition Method. Proceedings of the Conference of Applied Differential Geometry-General Relativity and the Workshop on Global Analysis, Differential Geometry and Lie Algebra, Balkan Press, 88-98.

[16] Adomian, G. (1989) Nonlinear Stochastic Systems Theory and Application to Physics. Kluwer Academic Publishers, Netherlands. http://dx.doi.org/10.1007/978-94-009-2569-4

[17] Bhadauria, R., Singh, A.K. and Singh, D.P. (2012) Solution of Reaction-Diffusion Equation by Adomian Decomposition Method. International Journal of Engineering Science and Technology, 4, 2626-2630. 